\title{
CONVEXITY OF THE FIELD \\ OF A LINEAR TRANSFORMATION
}

\section{A.J. Goldman and M. Marcus \\ (received June 16,1958 )}

Let $U_{n}$ be an $n$-dimensional unitary space with inner product $(x, y)=\overline{(y, x)} . \quad$ In $U_{n}$ let $S_{n-1}$ denote the unit sphere:

$$
s_{n-1}=\{x \mid(x, x)=1\} \text {. }
$$

Let $A$ be an arbitrary linear transformation of $U_{n}$. The subset

$$
\left.F(A)=\{\} \mid \zeta=(A x, x), x \text { in } S_{n-1}\right\}
$$

of the $\zeta$-plane $(\zeta=\xi+i \eta)$ is called the field of $A$.

As the image of $S_{n-1}$ under the continuous mapping $x \rightarrow(A x, x)$, F(A) must be compact and connected. Toeplitz proved in [4] that the boundary of $F(A)$ is a convex curve. Hausdorff then showed [2] that $F(A)$ actually fills the interior of this curve (i.e., that $F(A)$ is convex). Proofs of the convexity of $F(A)$ also appear in [3] and [5].

The purpose of this note is to provide a simple inductive proof for the convexity of $F(A)$ which reduces the essential computation to the single case $n=2$. We then dispose of this case by verifying directly that $F(A)$ satisfies the definition of a convex set.

THEOREM. $F(A)$ is convex.

Proof. (a) If $n=1$, then $F(A)$ is a single point.

(b) Deferring the case $n=2$, we suppose $n \geqslant 3$ and consider the inductive step from $n-1$ to $n$. Let $x$ and $y$ be any two vectors of $S_{n-1}$; we must show that $F(A)$ contains the segment joining the points $(A x, x)$ and $(A y, y)$ in the $\zeta$-plane. Since $n \geqslant 3$, we can find a vector $u$ in $U_{n} \operatorname{such}$ that $(u, x)=(u, y)=0$. The unitary-orthogonal complement in $U_{n}$ of the line $L$ spanned by $u$

Can. Math. Bull., vol.2, no. 1, Jan. 1959. 
is a subspace $U_{n-1}$ of $U_{n}$ whose unit sphere $S_{n-2}$ is contained in $\mathrm{S}_{\mathrm{n}-1}$; furthermore, $x$ and $y$ lie in $\mathrm{S}_{\mathrm{n}-2}$. Any vector $w$ in $\mathrm{U}_{n}$ admits a unique decomposition $w=v+z$, with $v$ in $L$ and $z$ in $U_{n-1}$; the unitary-orthogonal projection $P$ of $U_{n}$ onto $U_{n-1}$ is defined by $P w=z$. Obviously $A_{0}=P A P=P(A P)$ is a linear transformation of $U_{n-1}$ into itself. For any $z$ in $S_{n-2}$ (and thus in $S_{n-1}$ ) we have $\mathrm{Pz}=\mathrm{z}$ and thus, decomposing $\mathrm{Az}=\mathrm{v}_{1}+\mathrm{z}_{1}$,

$$
(A z, z)=\left(v_{1}+z_{1}, z\right)=\left(z_{1}, z\right)=(P A z, z)=(P A P z, z)=\left(A_{0} z, z\right) ;
$$

since $\left(A_{O} z, z\right)=(A z, z), F\left(A_{O}\right)$ is a subset of $F(A)$. Also, taking $z=x$ and $z=y$, we see that $(A x, x)$ and $(A y, y)$ are in $F\left(A_{0}\right)$; $F\left(A_{0}\right)$ is convex by hypothesis, and so the segment joining ( $A x, x$ ) and $(A y, y)$ lies in $F\left(A_{0}\right)$ and thus in $F(A)$, as desired.

(c) We turn now to the case $n=2$. It is well known (see [1], for example) that there exists a coordinate system (or equivalently, a basis) in $U_{2}$ with respect to which the matrix of A takes a "superdiagonal" form

$$
A=\left(\begin{array}{ll}
a & c \\
0 & b
\end{array}\right)
$$

so that for any vector $\mathrm{x}$ in the "unit circle" $S_{1}$ of $U_{2}$, with coordinates $x_{1}, x_{2}$ relative to the system, we have

$$
\begin{aligned}
(A x, x) & =a\left|x_{1}\right|^{2}+b\left|x_{2}\right|^{2}+c \bar{x}_{1} x_{2} \quad\left(\left|x_{1}\right|^{2}+\left|x_{2}\right|^{2}=1\right) \\
& =b+(a-b)\left|x_{1}\right|^{2}+c \bar{x}_{1} x_{2} .
\end{aligned}
$$

If, using the convention arg $(0)=0$, we let

$$
\begin{array}{ll}
\alpha=|a-b| & (\alpha \geqslant 0) \\
t=\arg (a-b) & (0 \leqslant s \leqslant 1) \\
s=\left|x_{1}\right|^{2} & \\
\theta=\operatorname{arg~} x_{2}-\operatorname{arg~x_{1}-t,} &
\end{array}
$$

and consider the set $S=[F(A)-b] \exp (-i t)$, we find that 


$$
s=\left\{\zeta \mid \zeta=\alpha s+c(s(1-s))^{\frac{1}{2}} \exp (i \theta) ; 0 \leqslant s \leqslant 1,0 \leqslant \theta \leqslant 2 \pi\right\} .
$$

Since $S$ is congruent to $F(A)$, it suffices to prove that $S$ is convex.

If $c=0$, then $S$ is a line segment and therefore convex. If $c \neq 0$ then we can assume $c=1$, since $F(A)$ is convex if and only if $c^{-1} F(A)=F\left(c^{-1} A\right)$ is convex. Thus we can take $S$ to be the union of the circles

$$
C(s): \quad|\zeta-\alpha s|=(s(1-s))^{\frac{1}{2}}=f(s) \quad(0 \leqslant s \leqslant 1) .
$$

Let $\zeta_{1}$ and $\zeta_{2}$ be any points of $S$ and let $\zeta_{0}$ be any point on the line joining them: we must show that 3 o lies in $S$. Let $C\left(s_{1}\right)$ and $C\left(s_{2}\right)$ be circles on which $\zeta_{1}, \zeta_{2}$ lie, and use the fact that jo can be written in the form

$$
3_{0}=r 3_{1}+(1-r) 3_{2} \quad(0 \leqslant r \leqslant 1)
$$

to define

$$
s_{0}=r s_{1}+(1-r) s_{2}
$$

Consider $G(s)=\left|\zeta_{0}-\alpha s\right|-f(s)$. Obviously $G(0)=\left|\zeta_{0}\right| \geqslant 0$ (i.e., $\zeta_{0}$ lies outside or on $C(0)$ ). We will show that $G\left(s_{0}\right) \leqslant 0$ (i.e., that $\zeta_{0}$ lies inside or on $\mathrm{C}\left(\mathrm{s}_{0}\right)$ ). It follows that $G\left(s^{*}\right)=0$ (i.e., that $\zeta_{0}$ lies on $C\left(s^{*}\right)$ ) for some $s^{*}$ with $0 \leqslant s * \leqslant s_{0} \leqslant 1$, so that $\zeta_{0}$ lies in $S$ and the convexity of $S$ will be proved.

To show that $G\left(s_{0}\right) \leqslant 0$, we apply the triangle inequality:

$$
\mid\}_{0}-\alpha s_{0}|\leqslant r| \beta_{0}-\alpha s_{1}|+(1-r)| \zeta_{0}-\alpha s_{2} \mid=r f\left(s_{1}\right)+(1-r) f\left(s_{2}\right) .
$$

Since $f^{\prime \prime}(s) \leqslant 0$ for $0<s<1$, we have

$$
r f\left(s_{1}\right)+(1-r) f\left(s_{2}\right) \leqslant f\left(s_{0}\right)
$$

and so $\left|\zeta_{0}-\alpha s_{o}\right| \leqslant f\left(s_{0}\right)\left(i_{.} e_{.}, G\left(s_{0}\right) \leqslant 0\right)$. This completes the proof. 


\section{REFERENCES}

1. P.R. Halmos, Finite dimensional vector spaces, Annals of Mathematics Studies Number 7,(Princeton, 1942), 83-84.

2. F. Hausdorff, Der Wertvorrat einer Bilinearform, Math. Zeit. 3(1919), 314-316.

3. M. H. Stone, Linear transformations in Hilbert space, A.M.S. Colloquium Publication XV, (1932), 131-133.

4. O. Toeplitz, Das algebraische Analogen zu einem Satze von Fejer, Math. Zeit. 2(1918), 187-197.

5. A Wintner, Spektraltheorie der unendlichen Matrizen, (Leipzig, 1929), 34-37.

National Bureau of Standards

and

University of British Columbia 